\title{
HLA Matching in Pediatric Stem Cell Transplantation
}

\author{
Matthias Eyrich ${ }^{a}$ Harald Schulze $^{b}$ \\ a University Children's Hospital, University Hospital Würzburg, Würzburg, Germany; ${ }^{b}$ Institute of Experimental \\ Biomedicine, Chair I, University Hospital Würzburg, Würzburg, Germany
}

\section{Keywords}

HLA matching · Stem cell transplantation · Donor selection

\begin{abstract}
For several malignant and nonmalignant disorders such as leukemias, lymphomas, or inborn errors of hematopoiesis, stem cell transplantation is the only curative option. Depending on the underlying cause of the disease, the conditioning regimens, source of the stem cells, and graft composition may vary. Possible stem cell donors are selected from databases considering existing major histocompatibility genes of the donor and the recipient. This is currently performed by matching human leukocyte antigen (HLA)-A, -B, and -C for class I, as well as HLA-DRB1 and -DQB1 for class II. Stem cell transplantation for nonmalignant disorders is a specialty of pediatrics. While algorithms for donor selection in these cases are generally similar, the objective of optimizing a possible graft-versus-leukemia effect is less important. In this article, we aim to provide an overview on the current methods for HLA typing and the algorithms for HLA matching. We also address ethical aspects regarding children and minors as stem cell donors.

(c) 2019 S. Karger AG, Basel
\end{abstract}

\section{Introduction}

Finding a suitable donor for a patient in need of a stem cell transplantation based on HLA matching seems to be a straightforward procedure. However, advances in our knowledge about the HLA system and new technical achievements necessitate a constant adaption of the search algorithms. Furthermore, the patient's age and a distinct spectrum of diseases in children impact the criteria for selecting the best available donor. This review article aims to provide some insight into the general algorithms for HLA matching and stem cell donor selection and to emphasize some relevant considerations regarding donor selection in pediatrics.

\section{General Guidelines for HLA Matching}

The major histocompatibility complex (MHC) describes a fundamental mechanism for recognizing and segregating self from non-self structures. MHC proteins are heterodimeric proteins with an interaction surface for T cells. The human MHC is referred to as the human leukocyte antigen (HLA). The underlying gene loci are located in close genetic vicinity on the short arm of chromosome 6 (6p21.3). HLA molecules are both polygenic and polymorphic, with a high sequence homology overall. These features provide a certain challenge to accurate sequencing.

HLA typing has a long history and undergone continuous development of technical and computational improvements. Currently, serological typing results are still present in certain databases (at least for certain loci), but minimal sequencing standards have been implemented. In general, laboratories providing full HLA typing have to be accredited by the American Society for Histocompatibility and Immunogenetics (ASHI), the European Federation for Immunogenetics (EFI; www.efiweb.eu),

\section{KARGER}

(C) 2019 S. Karger AG, Basel 
the United Kingdom Accreditation Service (UKAS) (www.ukas.com), or Clinical Pathology Accreditation (CPA). Typing definitions (i.e., low, intermediate, and high resolution) and result reporting should follow the official nomenclature [1]. General guidelines on HLA matching have recently been published in the guideline of the British Society for Histocompatibility and Immunogenetics (BHSI) [2].

Mismatches in HLA alleles can result in different immunological effects, depending on whether the mismatch is in the host-versus-graft or the graft-versus-host direction. Host-versus-graft reaction denotes the recognition of donor MHC molecules (or at least of unknown donor-specific peptide sequences presented by donor MHC) by recipient $\mathrm{T}$ cells. This reaction results in graft rejection and is of major importance in organ transplantation. In allogeneic stem cell transplantation, this reaction might be of relevance in the context of low-intensity conditioning; however, our knowledge about the antigens driving these reactions is very limited. In contrast, graft-versus-host reactions have been extensively investigated over the last decades. Mismatches of several HLA alleles between donor and host cause lethal graft-versushost disease, necessitating profound T-cell depletion of the graft (see section Haploidentical Donor Selection below). Yet, graft-versus-host reactions also occur in transplantations without obvious HLA mismatches. These cases (making up the vast majority of all cases of graftversus-host disease) are caused by differences in polymorphic peptides between donor and host. The list of well-characterized and -validated so-called minor histocompatibility antigens - which are generated by single nucleotide polymorphisms, indels, or gene deletions - is steadily growing and subject to ongoing research [3]. Unraveling the complexity of minor histocompatibility antigen differences between donor and host will be key to separate graft-versus-host disease from a positive graft-versus-leukemia effect.

Describing distinct HLA gene loci provides the lowest level of information and reflects whether the expressed genes belong to class I or class II. All transplantation-relevant genes are expressed codominantly. Currently, 3 class I loci (HLA-A, -B, and -C) and 2 class II loci (HLADRB1 and -DQB1) need to be analyzed. These 5 genes on the diploid human genome add up to 10 loci and finally consider a fully matched donor-recipient set as 10/10. Of note, more class I loci exist, and their gene products fulfill essential functions during embryogenesis: HLA-G and HLA-E (which are clustered as class Ib genes) are expressed on fetal tissue and trophoblasts, and their interaction with maternal CD94/NKG2 receptors allows that the non-self fetal tissue is not recognized by the mother's immune system, thereby maintaining immune tolerance during pregnancy.

HLA Matching in Pediatric Stem Cell

Transplantation
The chromosomal organization of the HLA gene cluster at one single locus and our limited knowledge of additional gene functions or regulation within this gene cluster emphasize that the mere selection of "fitting" alleles between donor and recipient (which finally translates into a 10/10 score) does not reflect the biological significance as found in identical siblings. To address this issue, algorithms and programs are in place not only to identify donors with identical single HLA alleles but also to ensure that these alleles are best selected as complete haplotypes [4]. This becomes even more relevant to recipients with poor coverage in stem cell donor databases, i.e., those with a rare or mixed ethnicity [5].

The HLA nomenclature has been repeatedly revised and constitutes a system of four fields, each separated by a colon. The first field often reflects the "classic" serological or "allelic" number, whereas the second field reflects the specific allelic variation that results in a defined amino acid sequence. The third field reports synonymous variants that will encode for the same protein, whereas the fourth field reflects alterations in noncoding regions [6]. One attempt to facilitate readability was to include immunologically equivalent groups $\left(\mathrm{A}^{*} 02: 01: 01 \mathrm{G}\right)$ that comprise several distinct genes or to state that the resulting protein (for the parts of the protein encoded by exons 2 and 3 ) is identical (HLA-A*02:01P).

Typical molecular biological approaches to HLA genotyping include (a) sequence-specific primers, which allow low-to-high resolution (i.e., $\mathrm{A}^{*} 02$ ); (b) sequence-specific oligonucleotides, which provide low-to-intermediate resolution (i.e., $\mathrm{A}^{*} 02 \mathrm{BNT}$ ); and (c) sequence-based typing, which will lead to high resolution $\left(\mathrm{A}^{*} 02: 02\right.$ or $\left.\mathrm{A}^{*} 02: 02: 01 \mathrm{G}\right)$.

When donor-derived material has arrived, confirmatory typing is usually performed by intermediate-resolution assays (sequence-specific oligonucleotides). Relevant to stem cell transplantation are the interaction domains of the MHC molecule with the T-cell receptor (TCR). An MHC class I molecule contains 8 exons and shares a stabilizing $\beta_{2}$-microglobulin light chain. The interaction domain to CD8-positive cytotoxic T cells is encoded by exons 2 and 3 . In contrast, MHC class II molecules are heterodimers with a rather hypomorphic $\beta$-chain. The interaction domain is thus effectively only encoded by exon 2 of each gene. Identical in this context of stem cell transplantation is the translation into the same amino acids derived from these exons. This antigen recognition site match represents the lowest standard for identity.

Next-generation sequencing has been adopted for HLA typing. Its development has been hampered by the highly polymorphic features of the HLA loci, with the addition of several pseudogenes. Overall short read lengths in second-generation sequencing have led to sequence 
ambiguities that did not allow defining whether two variants are in cis or in trans position, especially when a cosegregation analysis was impossible due to limited access to family members (parents and siblings). New approaches that are referred to as third-generation sequencing have bypassed and solved many of these problems [7]. Targeted sequencing allows inclusion of the complete gene sequence (including introns) rather than only the minimally required exons 2 and 3 .

\section{Null Alleles}

The detection of null alleles poses an additional challenge. If a null allele is not recognized (i.e., due to a stop codon in exon 1 or a mutation in the promoter region), the regular sequence derived from exons 2 and 3 might suggest normal surface expression. Thus, the mismatch between donor and recipient could result in graft failure or in an increased graft-versus-host disease. German consensus guidelines [8] require an analysis of the three most common null alleles when certain additional loci are detected: $A^{*} 24: 09 \mathrm{~N}$ when $\mathrm{B}^{*} 27$ or $\mathrm{B}^{*} 40$ is detected; $B^{*} 51: 11 \mathrm{~N}$ when the haplotype $A^{*} 02: 01 \& C^{*} 15: 02 / 15: 13$ \& DRB ${ }^{*} 04: 02$ is present. $\mathrm{C}^{*} 04: 09 \mathrm{~N}$ represents the most common null allele and needs to be tested for when $\mathrm{B}^{*} 44$ : 03 is present. However, additional null alleles might occur. As serotyping is hardly performed for stem cell transplantations, the presence of these HLA proteins on the cell surface is typically not tested. The new deep sequencing approaches might help to detect these null alleles.

\section{HLA-DP and HLA-DRB3/4/5}

Expression levels differ between certain HLA molecules: HLA-A, -B, -C, and -DR are usually expressed at a higher level than HLA-DQ or HLA-DP. The latter ones are also less polymorphic, so that the necessity of a match for these loci is less clear. Over the last decade, the association of HLA-DP matching with clinical outcome has been investigated extensively. Several reports have described that certain allele constellations are associated with increased T-cell cytotoxicity. Besides classic matching for HLA-DPB1, the group of Fleischhauer have developed the T-cell epitope (TCE) algorithm, which categorizes DP alleles into three (or four) groups of antigens that elicit similar T-cell reactivity $[9,10]$. The TCE model has been implemented in several search algorithms and is also included in the OptiMatch platform [11]. HLA-DRB3, -DRB4, and -DRB5 are genes that are only present in certain haplotypes and behave like alleles of a single locus. Their overall expression level is low [12]. Nevertheless, programs used in search units might provide sequencing results for these alleles too, and matching should be considered when appropriate, i.e., when a match or mismatch is obvious from the matching data.

\section{Predicted Indirectly ReCognizable HLA}

\section{Epitopes (PIRCHE)}

The TCE algorithm was developed based on elegant experiments which directly addressed the recognition of HLA epitopes by cytotoxic T cells. In contrast, the presentation of foreign, non-self antigens by MHC molecules formed the basis for a model called Predicted Indirectly ReCognizable HLA Epitopes (PIRCHE). In this model, relevant mismatches are considered for presentation, and scores for MHC class I and class II can be calculated. Studies have been made that clearly emphasize that the algorithms allow a functional correlation with the outcome $[13,14]$. The underlying algorithms are continuously improved [15]. These models might be of value to those patients for whom no fully matched donor is available in the databases.

For solid organ transplantation, the recipient immune system will detect and recognize histocompatibility complexes of the transplanted organ (host-versus-graft direction). A homozygous locus $\left(\mathrm{A}^{*} 02: 01 / \mathrm{A}^{*} 02: 01\right)$ will thus be perceived as identical even if the recipient has two different alleles $\left(A^{*} 02: 01 / A^{*} 03: 01\right)$ for this locus. For stem cell transplantation, the point of view is more complex, with a focus on the graft-versus-host direction. Discrepancies regarding homozygosity have to be considered for the graft-versus-host direction as well as for the host-versus-graft direction, especially when planning the intensity of the preparative regimen.

\section{The Search for a Suitable Donor}

Currently, three sources of donors may be considered: (1) related donors, (2) unrelated donors, and (3) cord blood units. An evolving body of evidence suggests that matching for HLA alleles is more important than the degree of relationship. At least in the pediatric field, results after allogeneic transplantation for hematologic malignancies with either matched related or unrelated donors are considered equivalent. Although for historical reasons there still is a preference for matched sibling donors (MSD), in case of an available matched unrelated donor (MURD) such donations should be limited to siblings with the ability to reason, i.e., those above the age of 14 years. With regard to nonmalignant disorders such as sickle cell disease, the outcome data still are more beneficial with matched related donors, even if data with alternative donors are evolving. In case no matched donor is available, mismatched related donors or cord blood units represent feasible options, with a worldwide trend towards haploidentical transplantations. In Germany, cord blood transplantations essentially are not performed anymore, but they are still more common in other countries, such as the USA and France, with larger cord blood banks. 
Thus, due to donor availability in daily clinical routine in developed countries, the transplant physician usually decides between a matched unrelated and a mismatched related donor, with a preference for the former.

\section{Related Donors}

Among related donors, the best choice is a full MSD. Such a donor matched for HLA class I and class II will typically also bring identity for class III genes that are situated between class I and class II genes. Identity for HLA$\mathrm{A},-\mathrm{B}$, and $-\mathrm{C}$ as well as for HLA-DRB1 and -DQB1 does not guarantee that all MHC are identical, as some crossing over might occur for those loci without a linkage disequilibrium, like HLA-DPB1.

A special situation arises with monozygotic twins. Here, not only are the donor and the recipient fully identical for all major and minor histocompatibility genes, but there is also an overall genetic identity. In this case, we expect no graft rejection or graft-versus-host disease; also, there will be no graft-versus-leukemia effect. Depending on the underlying medical indication for the hematopoietic stem cell transplantation, the monozygotic twin might be well suited or not. Of note, with increasing knowledge about epigenetic features, we know that relevant differences in protein expression might evolve, even between these otherwise identical siblings; however, our currently applied molecular sequencing approaches are blind in detecting these differences.

\section{Unrelated Donors}

Unrelated donors are now considered to be an excellent choice when they are fully matched (MURD). National and international databases have accumulated data on many potential donors, and for some patients, more than one fully matched donor is available. As the age of the donor has an important impact on the outcome, younger donors should be preferred over older donors, as well as over related donors. However, it is unclear whether this holds true for all donor-recipient constellations [16].

Besides HLA matching, additional criteria should be taken into consideration when selecting the best available donor. For instance, in haploidentical transplantations, using a female donor for a male recipient is associated with an increased risk of graft-versus-host disease [17], but this difference might also confer a better survival [18]; sensitization of a female donor with a history of multiple pregnancies to male minor histocompatibility antigens or HLA alleles is a potential mechanism for this phenomenon. In T-replete allotransplantation, the situation might be entirely different. Here, male donors have been shown to exhibit stronger T-cell reactivity against tumor-associated antigens than female donors [19], and female-tomale transplantations were associated with significantly reduced survival [20].
Virus reactivation poses a threat to immunocompromised patients; cytomegalovirus (CMV) and, to a lesser extent, adenoviruses and Epstein-Barr virus are the most relevant in this regard. Thus, CMV serostatus is a typical parameter; a $\mathrm{CMV}$-negative recipient should receive stem cells from a negative donor, and a positive recipient from a seropositive donor [21].

\section{Cord Blood Units}

Cord blood units are a further stem cell source; however, clinical experience with and preference for this graft type vary widely between countries. Hematopoietic progenitor cells present in cord blood provide unique features, with a more fetal signature overall. Since the success of the first cord blood transplantation in 1988, it has been assumed that less active or hypoactive $\mathrm{T}$ cells in the unit might explain why there generally is less graft-versus-host disease after cord blood transplantation than after bone marrow or peripheral blood stem cell transplantation. Currently, HLA typing is often limited to HLA-A, $-B$, and -DR, partly assuming that, due to linkage disequilibrium, HLA-C and -DQ will match in the majority of cases. The mismatch in one or two HLA loci allows fostering a graft-versus-leukemia effect without the risk of excessive graft-versus-host disease. This aspect is especially relevant with malignant indications; however, cord blood transplantation has also been applied to nonmalignant indications including immune deficiencies or hemoglobinopathies [reviewed in 22 in references therein].

One major hurdle is the limited amount of available material [23]. A very recent study [24] has evaluated over 126,000 cord blood units in the USA and found that many units contain fewer cells than originally thought. As a matter of fact, cord blood units might be a feasible option for smaller children; however, the available numbers of cells in cord blood grafts are problematic for full-grown adults. The authors concluded that only about half of the units were acceptable for patients weighing $30 \mathrm{~kg}$ and $30 \%$ for patients weighing $40 \mathrm{~kg}$; for adults weighing $80 \mathrm{~kg}$, only $15 \%$ of the units were acceptable [24]. This problem was addressed by the concept of "double-unit cord blood transplantations," with considerable success although the transplantation immunology is only incompletely understood, due to the fact that there is a dominant unit that will finally take over hematopoiesis [25]. New technologies include ex vivo cord blood expansion, which might evade the issue of limited material [26, 27].

\section{Haploidentical Donor Selection}

In case there is no identical matched related donor and a patient cannot wait until a suitable MURD is found, a haploidentical donor should be considered, which typically is a parent or sibling. This approach using readily available donors dramatically expands the pool of possible 
transplantation candidates and represents a good option for patients with high-risk diseases. Therefore, this transplant option has experienced steady growth rates, with impressive success over the last years, especially in East Asian countries with small unrelated donor registries [28].

Transplant regimens with a haploidentical donor require profound in vitro or in vivo T-cell depletion to counteract the increased risk of graft-versus-host disease due to HLA mismatching. In vitro T-cell depletion with the latest technologies, such as TCRab/CD19 depletion, is still a favorable option for graft manipulation in haploidentical transplantation, since patients need only minimal immunosuppression after transplantation and very promising outcomes have been reported with this technique [29]. In haploidentical transplants with ex vivo Tcell depletion, there are no reports that the type of HLA mismatch has any impact on the transplantation outcome. In these cases, donor selection criteria other than HLA matching might matter - e.g., KIR mismatching in myeloid malignancies [30], the general preference for male donors and donors of younger age [31], and matching for CMV IgG serostatus and the AB0 system. However, the algorithms rating these non-HLA-related selection criteria have not been validated in clinical trials so far.

Donor selection might differ in haploidentical transplantations with in vivo $\mathrm{T}$-cell depletion, e.g., using posttransplant cyclophosphamide or anti-thymocyte globulin. In this transplantation mode, one study group has identified an HLA-B mismatch as an independent risk factor for acute graft-versus-host disease [32], whereas this could not be confirmed by others $[31,33]$. Irrespective of the T-cell depletion method used, all recipients of a haploidentical stem cell transplant should be screened for donor-specific anti-HLA antibodies, as the presence of those is associated with primary graft failure and transplant-related mortality. Solid-phase approaches like the Luminex assays provide a reliable assay platform for detection of these relevant antibodies [34]. In case no donor-specific anti-HLA antibody-negative donor is available, inclusion of B-cell-depleting anti-CD20 antibodies (off label) and/or plasmapheresis in the preparative phase can help to minimize the risk of graft rejection or graft failure. Haploidentical stem cell transplantations open up new horizons in transplantation immunology, such as the discovery of the importance of noninherited maternal antigens [31] and HLA haplotype loss variants [35], giving the HLA system an entirely different significance.

\section{Ethical Considerations}

Over the last decades, HLA-matched children have been stem cell donors for their siblings in need of a stem cell transplantation. However, with more matched unrelated adult donors available in the national and international databases and the increasing equalization of MURD and MSD transplantations, it has become an ethical issue whether minors can or should be donating stem cells. The American Academy of Pediatrics provided a Policy Statement in 2010 with a series of 5 points that need to be fulfilled before a minor may be considered as a stem cell donor [36]. These discussions have been taken up in the UK [37]. The need of longitudinal observation and psychosocial assessment and management has now come into focus $[38,39]$. German policies have been set this year in Deutsches Ärzteblatt (DOI: 10.3238/arztebl.2019.rl_haematop_sz02).

One further step, if no HLA-matched donors are available, is certainly the creation of "savior children" who are designed (or selected) by preimplantation HLA matching. There is a growing literature with comments and ethical considerations regarding this topic [40-45], as to whether children should be considered as stem cell donors at all when suitable MURD are available. Studies demonstrating comparable outcomes after MURD and MSD allotransplantations have prompted these reflections.

Finally, ethical recommendations need to be provided for guidance on the handling of accidental findings as a result of targeted sequencing, e.g., explanations of the HLA-associated disease prevalence with respect to autoimmune diseases [46].

\section{Special Issues in Pediatrics}

In general, the algorithms for HLA matching and stem cell donor selection are the same for pediatric and adult recipients. However, in adults, the vast majority of indications are malignant disorders with the need to eradicate the malignant cells and therefore to maximally exploit a possible graft-versus-leukemia effect. In children, malignant disorders like leukemias and lymphomas are also a typical indication for transplantation, but there are also nonmalignant indications (inborn defects of red blood cells [i.e., hemoglobinopathies], inherited thrombocytopenia [i.e., congenital amegakaryocytic thrombocytopenia], general immune defects [i.e., Wiskott-Aldrich syndrome], or metabolic disorders [adrenoleukodystrophy]). In these cases, a graft-versus-leukemia effect is not necessary, a reduced-intensity conditioning regimen can be applied and a stable mixed donor-recipient chimerism might be enough to cure the disease.

There is evidence that donors best suited for recipients with nonmalignant diseases might differ from donors for patients with a malignant disease. For example, among thalassemia patients, allogeneic transplantations from a matched sibling and from a MURD have resulted in similarly good results [47]. In contrast, among sickle cell disease patients, the good clinical results obtained with MSD trans- 
plants [48] could not be reproduced with transplants from unrelated donors [49]; thus, MURD transplants are not recommended to patients with this disorder. Current clinical trials are investigating whether haploidentical transplantation with TCR $\beta / C D 19$-depleted grafts represents a valuable option for these patients. Generally, the number of clinical studies with pediatric patients and nonmalignant disorders is rather low, and further multicenter trials are warranted to obtain more reliable data on this issue.

\section{Acknowledgements}

The authors would like to thank Dr. Kremena Todorova for critical comments on the manuscript.

\section{Statement of Ethics}

The authors have no ethical conflicts to disclose.

\section{Disclosure Statement}

The authors have no conflicts of interest to declare.

\section{Funding Sources}

The authors had no relevant funding that affected this review article.

\section{References}

1 Nunes E, Heslop H, Fernandez-Vina $M$, Taves C, Wagenknecht DR, Eisenbrey AB, et al. Definitions of histocompatibility typing terms. Blood. 2011 Dec;118(23):e180-3.

2 Little AM, Green A, Harvey J, Hemmatpour S, Latham K, Marsh SG, et al. BSHI Guideline: HLA matching and donor selection for haematopoietic progenitor cell transplantation. Int J Immunogenet. 2016 Oct;43(5):263-86.

3 Griffioen M, van Bergen CA, Falkenburg JH. Autosomal Minor Histocompatibility Antigens: How Genetic Variants Create Diversity in Immune Targets. Front Immunol. 2016 Mar; 7:100.

4 Schäfer C, Schmidt AH, Sauter J. Hapl-o-Mat: open-source software for HLA haplotype frequency estimation from ambiguous and heterogeneous data. BMC Bioinformatics. 2017 May;18(1):284

5 Barker JN, Boughan K, Dahi PB, Devlin SM, Maloy MA, Naputo K, et al. Racial disparities in access to HLA-matched unrelated donor transplants: a prospective 1,312-patient analysis. Blood Adv. 2019 Apr;3(7):939-44.

6 Madden K, Chabot-Richards D. HLA testing in the molecular diagnostic laboratory. Virchows Arch. 2019 Feb;474(2):139-47.

7 Ambardar S, Gupta R, Trakroo D, Lal R, Vakhlu J. High Throughput Sequencing: An Overview of Sequencing Chemistry. Indian J Microbiol. 2016 Dec;56(4):394-404.

8 Müller C, Mytilineos J, Ottinger H, Arnold R, Bader P, Beelen D, et al. German Consensus 2013 on Immunogenetic Donor Selection Criteria in Allogeneic Stem Cell Transplantation. Transfusionsmedizin. 2014;4:190-6.

9 Zino E, Frumento G, Marktel S, Sormani MP, Ficara F, Di Terlizzi S, et al. A T-cell epitope encoded by a subset of HLA-DPB1 alleles determines nonpermissive mismatches for hematologic stem cell transplantation. Blood. 2004 Feb;103(4):1417-24.

10 Fleischhauer K, Shaw BE, Gooley T, Malkki M, Bardy P, Bignon JD, et al. Effect of T-cellepitope matching at HLA-DPB1 in recipients of unrelated-donor haemopoietic-cell trans- plantation: a retrospective study. Lancet Oncol. 2012 Apr;13(4):366-74.

11 Bochtler W, Beth M, Eberhard E, Müller C. OptiMatch $^{\circledR}$ - a universally configurable HLA matching framework. Tissue Antigens. 2008 Apr;71(4):321.

12 Fernández-Viña MA, Klein JP, Haagenson M, Spellman SR, Anasetti C, Noreen $\mathrm{H}$, et al. Multiple mismatches at the low expression HLA loci DP, DQ, and DRB3/4/5 associate with adverse outcomes in hematopoietic stem cell transplantation. Blood. 2013 May; 121 (22):4603-10.

13 Thus KA, Ruizendaal MT, de Hoop TA, Borst E, van Deutekom HW, Te Boome L, et al. Refinement of the definition of permissible HLA-DPB1 mismatches with predicted indirectly recognizable HLA-DPB1 epitopes. Biol Blood Marrow Transplant. 2014 Nov;20(11): 1705-10.

14 Thus KA, de Hoop TA, de Weger RA, Bierings MB, Boelens JJ, Spierings E. Predicted Indirectly ReCognizable HLA Epitopes Class I Promote Antileukemia Responses after Cord Blood Transplantation: Indications for a Potential Novel Donor Selection Tool. Biol Blood Marrow Transplant. 2016 Jan;22(1): 170-3.

15 Geneugelijk K, Spierings E. Matching donor and recipient based on predicted indirectly recognizable human leucocyte antigen epitopes. Int J Immunogenet. 2018 Apr;45(2):4153.

16 Kollman C, Spellman SR, Zhang MJ, Hassebroek A, Anasetti C, Antin JH, et al. The effect of donor characteristics on survival after unrelated donor transplantation for hematologic malignancy. Blood. 2016 Jan;127(2):260-7.

17 Gratwohl A, Passweg J, Baldomero H, Hermans J; European Group for Blood and Marrow Transplantation (EBMT). Blood and marrow transplantation activity in Europe 1996. Bone Marrow Transplant. 1998 Aug; 22(3):227-40.

18 Stern M, Ruggeri L, Mancusi A, Bernardo $\mathrm{ME}$, de Angelis C, Bucher C, et al. Survival after T cell-depleted haploidentical stem cell transplantation is improved using the mother as donor. Blood. 2008 Oct;112(7):2990-5.

19 Lutz M, Worschech A, Alb M, Gahn S, Bernhard L, Schwab M, et al. Boost and loss of immune responses against tumor-associated antigens in the course of pregnancy as a model for allogeneic immunotherapy. Blood. 2015 Jan;125(2):261-72.

20 Solh M, Zhang X, Connor K, Brown S, Morris LE, Holland HK, et al. Donor Type and Disease Risk Predict the Success of Allogeneic Hematopoietic Cell Transplantation: A SingleCenter Analysis of 613 Adult Hematopoietic Cell Transplantation Recipients Using a Modified Composite Endpoint. Biol Blood Marrow Transplant. 2017 Dec;23(12):2192-8.

21 Ljungman P. The role of cytomegalovirus serostatus on outcome of hematopoietic stem cell transplantation. Curr Opin Hematol. 2014 Nov;21(6):466-9.

22 Munoz J, Shah N, Rezvani K, Hosing C, Bollard CM, Oran B, et al. Concise review: umbilical cord blood transplantation: past, present, and future. Stem Cells Transl Med. 2014 Dec;3(12):1435-43.

23 Querol S, Rubinstein P, Marsh SG, Goldman J, Madrigal JA. Cord blood banking: 'providing cord blood banking for a nation'. Br J Haematol. 2009 Oct;147(2):227-35.

24 Barker JN, Kempenich J, Kurtzberg J, Brunstein CG, Delaney C, Milano F, et al. CD34+ cell content of 126,341 cord blood units in the US inventory: implications for transplantation and banking. Blood Adv. 2019 Apr;3(8):1267-71.

25 Gutman JA, Riddell SR, McGoldrick S, Delaney C. Double unit cord blood transplantation: who wins - and why do we care? Chimerism. 2010 Jul-Sep;1(1):21-2.

26 Wagner JE Jr, Brunstein CG, Boitano AE, DeFor TE, McKenna D, Sumstad D, et al. Phase I/II Trial of StemRegenin-1 Expanded Umbilical Cord Blood Hematopoietic Stem Cells Supports Testing as a Stand-Alone Graft. Cell Stem Cell. 2016 Jan;18(1):144-55. 
27 Horwitz ME, Wease S, Blackwell B, Valcarcel D, Frassoni F, Boelens JJ, et al. Phase I/II Study of Stem-Cell Transplantation Using a Single Cord Blood Unit Expanded ex vivo with Nicotinamide. J Clin Oncol. 2019 Feb; 37(5):367-74.

28 Niederwieser D, Baldomero H, Szer J, Gratwohl M, Aljurf M, Atsuta Y, et al. Hematopoietic stem cell transplantation activity worldwide in 2012 and a SWOT analysis of the Worldwide Network for Blood and Marrow Transplantation Group including the global survey. Bone Marrow Transplant. 2016 Jun; 51(6):778-85.

29 Lang P, Handgretinger R, Meisel R, Mielke S, Niederwieser D, Schlegel P, et al. Results of a Prospective, Multicenter, Phase 1/ll Clinical Study in Pediatric and Adult Patients Using TCR Alpha/Beta and CD19 Depleted Haploidentical Hematopoietic Stem Cell Grafts following Reduced-Intensity Conditioning. Blood. 2018;132 Suppl 1:604.

30 Heidenreich S, Kröger N. Reduction of Relapse after Unrelated Donor Stem Cell Transplantation by KIR-Based Graft Selection. Front Immunol. $2017 \mathrm{Feb}$;8:41.

31 Wang Y, Chang YJ, Xu LP, Liu KY, Liu DH, Zhang XH, et al. Who is the best donor for a related HLA haplotype-mismatched transplant? Blood. 2014 Aug;124(6):843-50.

32 Huo MR, Xu LP, Li D, Liu DH, Liu KY, Chen $\mathrm{H}$, et al. The effect of HLA disparity on clinical outcome after HLA-haploidentical blood and marrow transplantation. Clin Transplant. 2012 Mar-Apr;26(2):284-91.

33 Wang M, Dong YJ, Qiu ZX, Wang MJ, Liu W, Wang LH, et al. HLA disparity is not crucial for the survival rate and severity of chronic health conditions in adult recipients following family donor hematopoietic stem cell transplantation. Int J Hematol. 2015 Jan; 101(1):75-82.
34 Lachmann N, Todorova K, Schulze H, Schönemann C. Luminex ${ }^{\circledR}$ and its applications for solid organ transplantation, hematopoietic stem cell transplantation, and transfusion. Transfus Med Hemother. 2013 Jun; 40(3):182-9.

35 Villalobos IB, Takahashi Y, Akatsuka Y, Muramatsu $\mathrm{H}$, Nishio $\mathrm{N}$, Hama A, et al. Relapse of leukemia with loss of mismatched HLA resulting from uniparental disomy after haploidentical hematopoietic stem cell transplantation. Blood. 2010 Apr;115(15):3158-61.

36 American Academy of Pediatrics. Committee on Bioethics. Children as hematopoietic stem cell donors. Pediatrics. 2010 Feb;125(2):392404.

37 Chan TK, Tipoe GL. The policy statement of the American Academy of Pediatrics - children as hematopoietic stem cell donors - a proposal of modifications for application in the UK. BMC Med Ethics. 2013 Oct; 14:43.

38 Pelletier W, Schulte F, Guilcher GM. Pediatric hematopoietic stem cell donors: need for longitudinal medical and psychosocial surveillance. Pediatr Blood Cancer. 2015 May;62(5): 737-8.

39 Wiener L, Hoag JA, Pelletier W, Shah NN, Shaw BE, Pulsipher MA, et al. Transplant center practices for psychosocial assessment and management of pediatric hematopoietic stem cell donors. Bone Marrow Transplant. 2019 [Epub ahead of print].

40 Devolder K. Preimplantation HLA typing: having children to save our loved ones. J Med Ethics. 2005 Oct;31(10):582-6.

41 Samuel GN, Strong KA, Kerridge I, Jordens CF, Ankeny RA, Shaw PJ. Establishing the role of pre-implantation genetic diagnosis with human leucocyte antigen typing: what place do "saviour siblings" have in paediatric transplantation? Arch Dis Child. 2009 Apr; 94(4):317-20
42 Hui EC, Chan C, Liu A, Chow K. Attitudes of Chinese couples in Hong Kong regarding using preimplantation genetic diagnosis (PGD) and human leukocyte antigens (HLA) typing to conceive a 'Saviour Child'. Prenat Diagn. 2009 Jun;29(6):593-605.

43 Burgio GR, Nespoli L, Maccario R, Verri A, Comoli P, Zecca M. Conceiving a hematopoietic stem cell donor: twenty-five years after our decision to save a child. Haematologica. 2012 Apr;97(4):479-81.

44 Taylor-Sands M. Summary of Saviour Siblings. J Med Ethics. 2015 Dec;41(12):926.

45 Kakourou G, Vrettou C, Moutafi M, TraegerSynodinos J. Pre-implantation HLA matching: the production of a Saviour Child. Best Pract Res Clin Obstet Gynaecol. 2017 Oct;44: 76-89.

46 Dendrou CA, Petersen J, Rossjohn J, Fugger L. HLA variation and disease. Nat Rev Immunol. 2018 May; 18(5):325-39.

47 Huang K, Zhou DH, Li Y, Xu HG, Que LP, $\mathrm{Chen} \mathrm{C}$, et al. Modified conditioning regimen improves outcomes of unrelated donor peripheral blood stem cell transplantation for $\beta$-thalassaemia major patients. Pediatr Blood Cancer. 2018 Jul;65(7):e27026.

48 Gluckman E, Cappelli B, Bernaudin F, Labopin M, Volt F, Carreras J, et al. Sickle cell disease: an international survey of results of HLA-identical sibling hematopoietic stem cell transplantation. Blood. 2017 Mar;129(11): 1548-56.

49 Shenoy S, Eapen M, Panepinto JA, Logan BR, Wu J, Abraham A, et al. A trial of unrelated donor marrow transplantation for children with severe sickle cell disease. Blood. 2016 Nov;128(21):2561-7. 